\title{
On the correlation between electric polarizabilities and the ionization potential of atoms
}

\author{
B. Fricke \\ Department of Physics, University of Kassel, D-3500 Kassel, West Germany
}

(Received 15 May 1985; accepted 1 August 1985)

It is found that the electric dipole polarizabilities of neutral atoms correlate very strongly with their first ionization potential within the groups of elements with the same angular momenta of the outermost electrons. As the latter values are known very accurately, this allows a very good $(<30 \%)$ prediction of various atomic polarizabilities.

\section{INTRODUCTION}

The electric dipole polarizability describes the response in lowest order of the field strength of the electron cloud to an electric field. This property plays an important role in a large number of physical quantities and processes. The review of Miller and Bederson ${ }^{1}$ describes in great detail the methods of calculation as well as the experimental measurements of the electric dipole polarizability $\alpha$. In the Appendix they list the recommended values for this quantity for all atoms throughout the periodic system. This Appendix shows only 32 values with an estimated accuracy of $8 \%$ or better, whereas the remaining ones are listed with an estimated accuracy of only $50 \%$. This still presents a great challenge to experimentalists as well as theoreticians.

Just recently, a paper by Kolb et $a l^{2}{ }^{2}$ was published in which electric and magnetic susceptibilities and shielding factors for closed-shell atoms and ions of high nuclear charges are calculated with the relativistic random phase approximation (RRPA) method. This method, as well as those described in the article by Miller and Bederson, ${ }^{1}$ are very laborious, and the comparison with experimental values-in themselves very hard to measure-is still not very satisfactory.
As the electric dipole polarizability is a measure for the response of an atomic system to an external electric field, and the first ionization potential measures the extraction energy of the outermost electron of the atom, we tried to correlate both quantities. In Fig. 1 we present the first ionization potential of all atoms vs the electric dipole polarizability in a double-logarithmic scale. The values (taken from Ref. 1) with an estimated accuracy of $8 \%$ or better are presented as crosses, and all the others just as points. A general trend appears showing at least the existence of some correlation of these two quantities. Only a very small number of points are relatively far off.

Of course, both quantities cannot be expected to correlate strongly in such a general way for all elements, because the structure of the valence electrons of each element is very different. But it is well known from the periodic system that the elements are structured in chemical groups of very analogous behavior with an analog structure of the outer electrons. And as both quantities, the ionization potential as well as the electric polarizability, also depend very much on the specific structure of the outer electrons, it is reasonable to assume that they correlate somehow. To verify whether this supposition could help to predict better values for the polar-

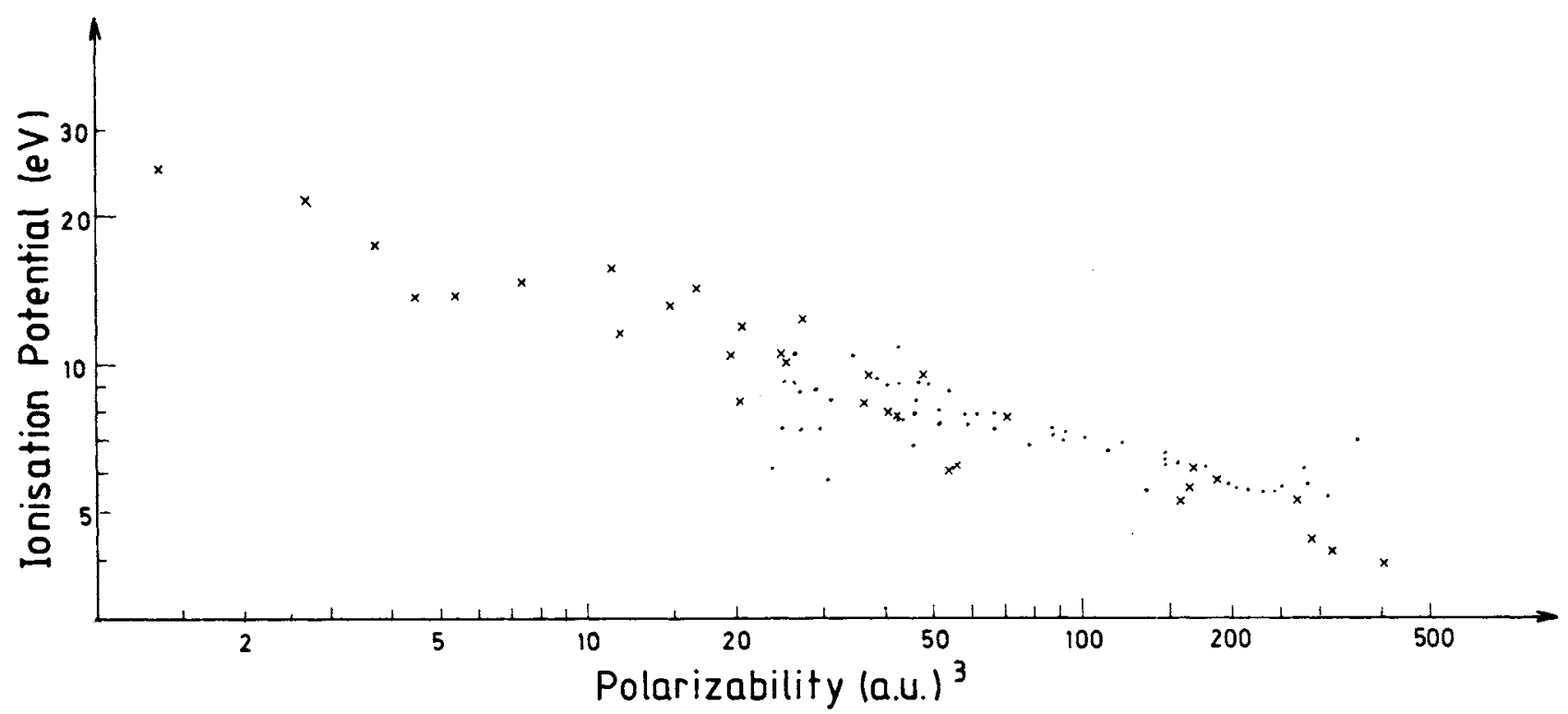

FIG. 1. First ionization potential vs the electric dipole polarizability $\alpha$ of all elements in a log-log scale. The values of $\alpha$ are taken from Ref. 1 . The crosses have an estimated accuracy of $\leqslant 8 \%$; all other points have a $50 \%$ estimated accuracy. 


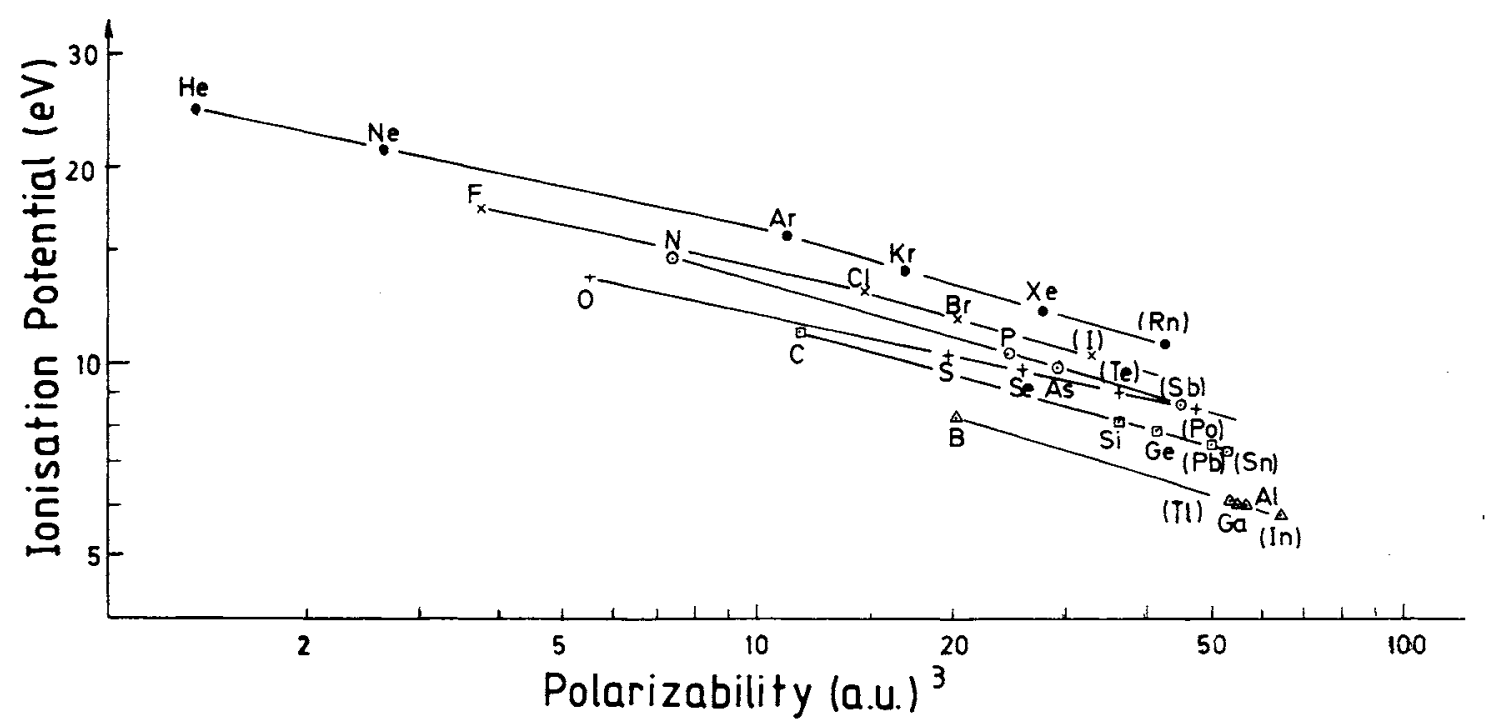

FIG. 2. Same plot as in Fig. 1 for the $2 p$ to $6 p$ elements. Connected are the elements of the same chemical group. The elements in brackets are extrapolated values.

izability we present in Figs. 2-7 the ionization potential vs the electric dipole polarizability $\alpha$ for the different $p, d$, and $s$ electron series of the periodic system.

\section{POLARIZABILITIES FOR THE $p$ ELEMENTS}

Figures 2 and 3 present the elements of the $2 p$ to the $6 p$ series. The elements with no brackets are those known with an estimated accuracy of $2 \%$ or better (according to Ref. 1). In Fig. 2 we connect all elements of the same chemical groups (which have the same symbol) with each other. It is remarkable here that the elements of the same chemical groups are aligned on almost perfectly straight lines. Only at the $3 p$ elements a very small angle appears in these lines. The reason is probably that the $4 p$ and $5 p$ elements have underlying $3 d, 4 d$ electron shells, with a resulting different behavior of the $4 p$ to $6 p$ elements in comparison to the $2 p$ and $3 p$ elements. The connecting lines run surprisingly parallel, both for the first ( $2 p$ to $3 p$ elements) as well as the second part ( $3 p$ to $6 p$ elements). This straight line behavior allows a prediction of the polarizabilities of those elements known up to $50 \%$ accuracy only. The values for the $5 p$ elements $\mathrm{Rn}, \mathrm{I}, \mathrm{Te}$, $\mathrm{Sb}, \mathrm{Sn}$, and In are deduced from an extrapolation of the lines in Fig. 2 by just using their well known first ionization potential.

This prediction can be cross checked: The same elements are presented in Fig. 3. This time, however, they are connected within the same main quantum number. Here again the first and last three of these elements within each $p$ group are connected by straight lines. The break between the third and fourth element is due to the change of the trend of the ionization potential because of the Hunds rule. This can be seen for the $2 p, 3 p$, and $4 p$ elements.

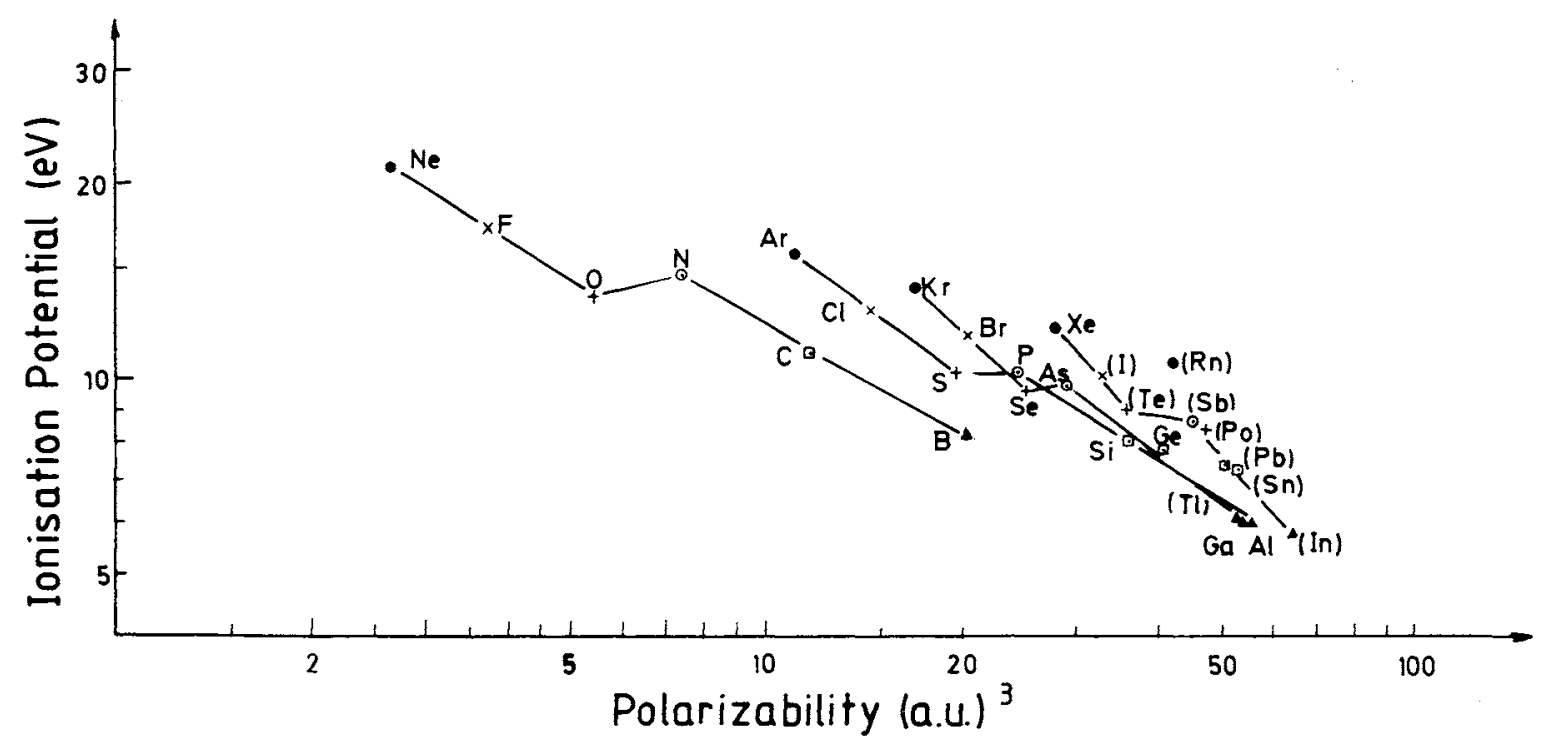

FIG. 3. Same plot as in Fig. 2 for the $2 p$ to $6 p$ elements. Connected are the elements of the same $n p$ elements from $2 p$ to the $5 p$ elements. 


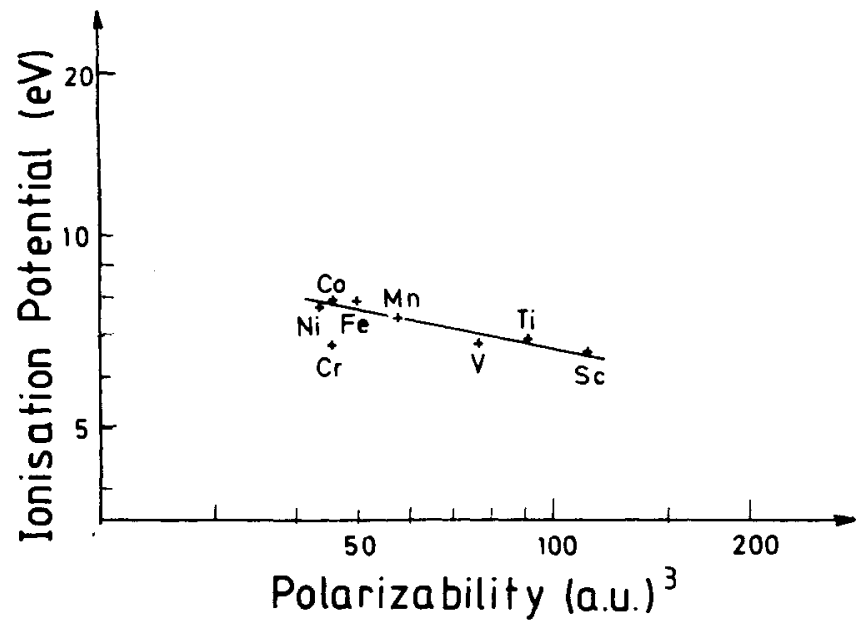

FIG. 4. Same plot as in Fig. 1 for the $4 d$ elements. The values of $\alpha$ are taken from Ref. 1.

If we use the values for the $5 p$ elements-predicted by the extrapolation in Fig. 2-we obtain again two straight lines for $\mathrm{Xe}, \mathrm{I}, \mathrm{Te}$, and $\mathrm{Sb}, \mathrm{Sn}, \mathrm{In}$. The break between $\mathrm{Te}$ and $\mathrm{Sb}$ is consistent with that in the $2 p$ to $4 p$ elements. This general trend shows the consistency of the extrapolation, and allows to say that the extrapolated values of the polarizability for the $5 p$ elements are reasonably reliable. In Table I we are, therefore, listing the new values, and allow for an estimated error of less than $20 \%$.

The continuation to the $6 p$ elements is more complicated: First the ionization potential of At is not known, and secondly the break in the trend of the ionization potential arises between the second $(\mathrm{Pb})$ and third element $(\mathrm{Bi})$ of the $6 p$ series. This behavior of the ionization potential is well known, and can be explained by the breakdown of LS coupling, and the onset of the relativistic $j-j$ coupled $6 p_{1 / 2}$ and $6 p_{3 / 2}$ electron shells. If we extrapolate the values for the polarizability for the $6 p$ elements, according to the scheme in Fig. 2, the value for the element Bi is relatively far off. It is not possible to draw a straight line according to the scheme in Fig. 3 for this element $\mathrm{Bi}$. This behavior is probably due to the relativistic influence on the outer shell of $\mathrm{Bi}$. At $\mathrm{Pb}$ the

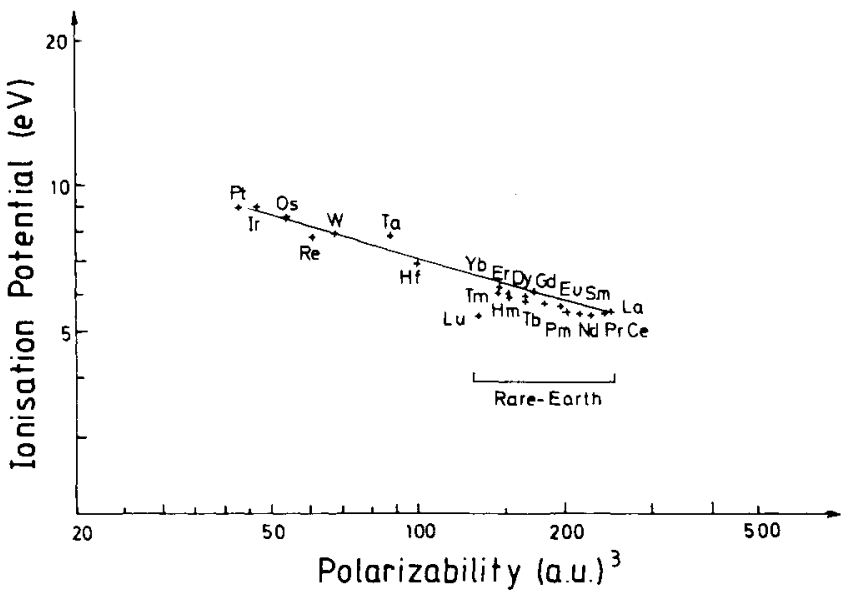

FIG. 6. Same plot as in Fig. 1 for the $6 d$ and $4 f$ elements. The values of $\alpha$ are taken from Ref. 1.

$6 p_{1 / 2}$ shell is complete with two electrons, and at Bi one electron is built into the $6 p_{3 / 2}$ shell. Thus Bi behaves more like an element with one electron in the outer shell. This argument of relativistic influences being strong applies to all the $6 p$ elements. Therefore, a straightforward extrapolation according to the scheme in Fig. 2, and consistent with the scheme of Fig. 3, is not possible for Bi. All the other $6 p$ elements are somewhere around 50 a.u. ${ }^{3}$ for the polarizability. Nevertheless, this is probably more realistic than the values between 25 and 35 a.u. ${ }^{3}$ given in Ref. 1.

\section{POLARIZABILITIES FOR THE $d$ ELEMENTS}

Figures 4-6 show the correlation between the first ionization potential and the polarizability for the $3 d, 4 d$, and $5 d$ elements, respectively. In Fig. 6 we included the values for the $4 f$ elements as well. In these figures the $\alpha$ values given in Ref. 1 are used. Again the values for the $d$ elements align on a straight line. Of the $3 d$ elements (Fig. 4) only the element $\mathrm{Cr}$ is relatively far off. As this is the only such occurrence for all the $3 d, 4 d$, and $5 d$ elements this value is probably a wrong one, although one would expect a little bit more structure because the ground states of the $d$ elements have outer va-

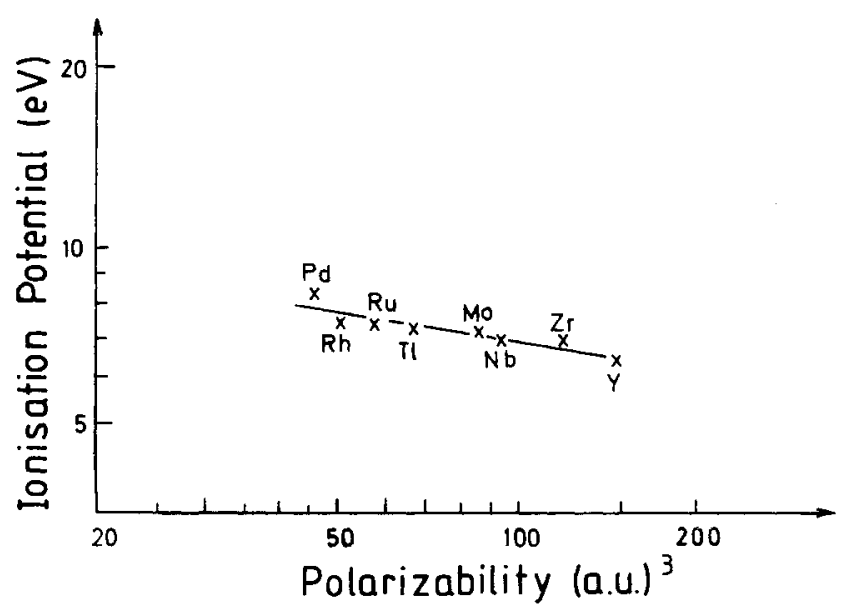

FIG. 5. Same plot as in Fig. 1 for the $5 d$ elements. The values of $\alpha$ are taken from Ref. 1.

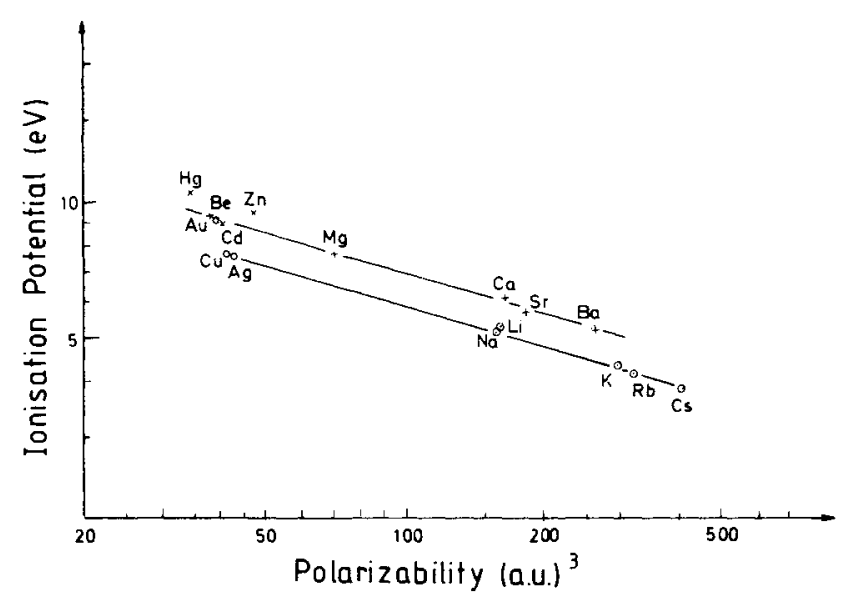

FIG. 7. Same plot as in Fig. 1 for the chemical groups Ia, IIa and Ib, IIb. The values are taken from Ref. 1 . 
lence shells containing two, one, or zero $s$ electrons. Table I lists the values for the polarizabilities taken from the straight line behavior of Figs. 4-6. The estimated accuracy of all these elements being $50 \%$ according to Ref. 1, these new values given in Table I can be expected to be better estimations than the recommended values of Ref. 1 .

Within the $4 f$ elements only Lu is off the line. As this element has a full $4 f^{14}$ shell, and contains a single $5 d$ electron as well, it is very similar to La. The ionization potential is very similar to $\mathrm{La}$, too. Therefore, it is consistent to change the value of $\alpha$ for this element to the much larger value given in Table I.

\section{POLARIZABILITIES FOR THE $s$ ELEMENTS}

Figure 7 shows the values for the groups Ia and IIa, as well as Ib and IIb elements. Again the elements align along nearly perfect straight lines which also run very parallel. The $\mathrm{Ib}$ elements $\mathrm{Cu}$ and $\mathrm{Ag}$ also lie on the same line as the Ia elements. Only $\mathrm{Au}$ is relatively far off. The IIb elements $\mathrm{Zn}$, $\mathrm{Cd}$, and $\mathrm{Hg}$ also follow more or less the straight line of the group IIa elements.

The values for $\alpha$ used in Fig. 7 are taken from Ref. 1 where all Ia and IIa elements have $\leqslant 8 \%$, and the Ib and IIb elements $50 \%$ estimated accuracy. Table I presents the extrapolated values for these elements. For the heavy elements $\mathrm{Hg}$ and $\mathrm{Au}$ we estimate a larger uncertainty than for the lighter ones, because it is not quite clear how relativity influences the values of $\alpha$ for the heavier elements.

\section{SUMMARY}

Figures 2-7 show the existence of a very strong correlation between the first ionization potential and the electric

TABLE I. First double column lists the recommended values of the atomic polarizabilities $\alpha$ in (a.u. ${ }^{3}$ ) plus the estimated accuracy from Ref. 1. Second double column lists the values for $\alpha$ as extrapolated from this work together with a new estimation of the accuracy.

\begin{tabular}{|c|c|c|c|c|c|c|c|c|c|}
\hline \multirow{2}{*}{ Elements } & Polarizability & $\begin{array}{c}\text { Estimated } \\
\text { accuracy }(\%)\end{array}$ & Polarizability & $\begin{array}{c}\text { Estimated } \\
\text { accuracy }(\%)\end{array}$ & \multirow{2}{*}{ Elements } & Polarizability & $\begin{array}{c}\text { Estimated } \\
\text { accuracy }(\%)\end{array}$ & Polarizability & $\begin{array}{c}\text { Estimated } \\
\text { accuracy }(\%)\end{array}$ \\
\hline & \multicolumn{2}{|c|}{ (From Ref. 1) } & \multicolumn{2}{|c|}{ (This work) } & & \multicolumn{2}{|c|}{ (From Ref: 1 ) } & \multicolumn{2}{|c|}{ (This work) } \\
\hline $\mathrm{H}$ & 4.5 & "Exact" & & & $\mathrm{Rh}$ & 51 & 50 & 60 & 20 \\
\hline $\mathrm{He}$ & 1.383 & "Exact" & & & $\mathrm{Pd}$ & 47 & 50 & 32 & 20 \\
\hline $\mathrm{Li}$ & 164 & 0.5 & & & $\mathrm{Ag}$ & 43 & 50 & 42 & 20 \\
\hline $\mathrm{Be}$ & 37.8 & 2 & & & $\mathrm{Cd}$ & 40 & 50 & 42 & 20 \\
\hline B & 20.4 & 2 & & & In & 30 & 50 & 64 & 10 \\
\hline $\mathrm{C}$ & 11.9 & 2 & & & $\mathrm{Sn}$ & 30 & 50 & 54 & 10 \\
\hline $\mathbf{N}$ & 7.42 & 2 & & & $\mathrm{Sb}$ & 27 & 50 & 46 & 10 \\
\hline 0 & 5.41 & 2 & & & $\mathrm{Te}$ & 26 & 50 & 36 & 10 \\
\hline $\mathbf{F}$ & 3.76 & 2 & & & I & 26 & 50 & 33 & 10 \\
\hline $\mathrm{Ne}$ & 2.67 & 2 & & & $\mathrm{Xe}$ & 27 & 0.5 & & \\
\hline $\mathrm{Na}$ & 159.3 & 2 & & & Cs & 402 & 2 & & \\
\hline $\mathrm{Mg}$ & 71.5 & 2 & & & $\mathrm{Ba}$ & 268 & 8 & 268 & 2 \\
\hline $\mathrm{Al}$ & 56.3 & 2 & & & $\mathbf{L a}$ & 250 & 50 & 250 & 30 \\
\hline $\mathrm{Si}$ & 36.3 & 2 & & & $\mathrm{Ce}$ & 243 & 50 & 260 & 30 \\
\hline $\mathbf{P}$ & 24.5 & 2 & & & $\mathrm{Pr}$ & 229 & 50 & 265 & 30 \\
\hline $\mathbf{S}$ & 19.8 & 2 & & & $\mathrm{Nd}$ & 216 & 50 & 260 & 30 \\
\hline $\mathrm{Cl}$ & 14.7 & 2 & & & $\mathrm{Pm}$ & 202 & 50 & 250 & 30 \\
\hline Ar & 11.1 & 0.5 & & & $\mathrm{Sm}$ & 196 & 50 & 235 & 30 \\
\hline $\mathrm{K}$ & 293 & 2 & & & $\mathbf{E u}$ & 182 & 50 & 230 & 30 \\
\hline $\mathrm{Ca}$ & 168 & 8 & & & $\mathrm{Gd}$ & 175 & 50 & 175 & 30 \\
\hline Sc & 114 & 50 & 110 & 20 & $\mathrm{~Tb}$ & 169 & 50 & 205 & 30 \\
\hline $\mathrm{Ti}$ & 92 & 50 & 86 & 20 & Dy & 169 & 50 & 190 & 30 \\
\hline V & 77 & 50 & 90 & 20 & Ho & 155 & 50 & 185 & 30 \\
\hline $\mathrm{Cr}$ & 46 & 50 & 90 & 20 & $\mathrm{Er}$ & 155 & 50 & 170 & 30 \\
\hline Mn & 58 & 50 & 58 & 20 & $\mathrm{Tm}$ & 148 & 50 & 170 & 30 \\
\hline $\mathrm{Fe}$ & 51 & 50 & 45 & 20 & $\mathrm{Yb}$ & 148 & 50 & 160 & 30 \\
\hline $\mathrm{Co}$ & 46 & 50 & 45 & 20 & $\mathbf{L u}$ & 135 & 50 & 260 & 30 \\
\hline $\mathrm{Ni}$ & 44 & 50 & 48 & 20 & Hf & 101 & 50 & 110 & 30 \\
\hline $\mathrm{Cu}$ & 41 & 50 & 41 & 20 & $\mathrm{Ta}$ & 88 & 50 & 74 & 30 \\
\hline $\mathrm{Zn}$ & 47.8 & 2 & 37 & 20 & W & 67 & 50 & 67 & 30 \\
\hline $\mathrm{Ga}$ & 54.8 & 2 & & & $\operatorname{Re}$ & 61 & 50 & 72 & 30 \\
\hline $\mathrm{Ge}$ & 41.0 & 2 & & & Os & 54 & 50 & 52 & 30 \\
\hline As & 29.1 & 2 & & & Ir & 47 & 50 & 44 & 30 \\
\hline Sc & 25.4 & 2 & & & $\mathbf{P t}$ & 43 & 50 & 45 & 30 \\
\hline $\mathrm{Br}$ & 20.6 & 2 & & & $\mathrm{Au}$ & 38 & 50 & 25 & 30 \\
\hline $\mathrm{Kr}$ & 16.7 & 0.5 & & & $\mathbf{H g}$ & 34 & 50 & 26 & 30 \\
\hline $\mathrm{Rb}$ & 319 & 2 & & & $\mathrm{Tl}$ & 24 & 50 & 54 & 30 \\
\hline $\mathrm{Sr}$ & 186 & 8 & 195 & 8 & $\mathrm{~Pb}$ & 25 & 50 & 50 & 30 \\
\hline $\mathrm{Y}$ & 148 & 50 & 148 & 20 & $\mathrm{Bi}$ & 27 & 50 & 50 & 50 \\
\hline $\mathrm{Zr}$ & 121 & 50 & 105 & 20 & Po & 31 & 50 & 48 & 30 \\
\hline $\mathrm{Nb}$ & 94 & 50 & 95 & 20 & At & 34 & 50 & 46 & 50 \\
\hline Mo & 88 & 50 & 80 & 20 & $\mathrm{Rn}$ & 43 & 50 & 42.5 & 20 \\
\hline $\mathrm{Tc}$ & 67 & 50 & 70 & 20 & $\mathrm{Fr}$ & 452 & 50 & & \\
\hline $\mathrm{Ru}$ & 58 & 50 & 68 & 20 & $\mathrm{Ra}$ & 310 & 50 & 250 & 20 \\
\hline
\end{tabular}


dipole polarizability when differentiating for the various chemical groups. We find a very good straight line behavior in a $\log -\log$ plot for nearly all elements of each group.

Table I lists the recommended values by Miller and Bederson ${ }^{1}$ as well as their estimated accuracy in the first double column. In the second double column we summarize those values obtained by the extrapolation according to the scheme discussed here. We list only those values which are different compared to the list in Ref. 1 or where we estimate a different error.

We believe the error estimate of $50 \%$ of Miller and Bederson ${ }^{1}$ to be generally too large as most values form a very straight line. Of course, all the extrapolated values will change when the fix points change, but we still think that the error estimate of $20 \%-30 \%$ for most of the higher $Z$ elements is sufficient to take this into account.

Naturally, this discussion is nothing else but an empirical extrapolation where the well known values for the ionization potentials are used via the straight line correlation to obtain better values for the electric dipole polarizability. It will be interesting to see whether further experimental and theoretical determinations justify this procedure.

${ }^{1}$ T. H. Miller and B. Bederson, Adv. At. Mol. Phys. 13, 1 (1977).

${ }^{2}$ D. Kolb, W. R. Johnson, and P. Shorer, Phys. Rev. A 26, 19 (1982). 